\title{
Endoscopic management of a rare cause of upper gastrointestinal bleeding: gastric polypoid extra- medullary hemopoiesis
}

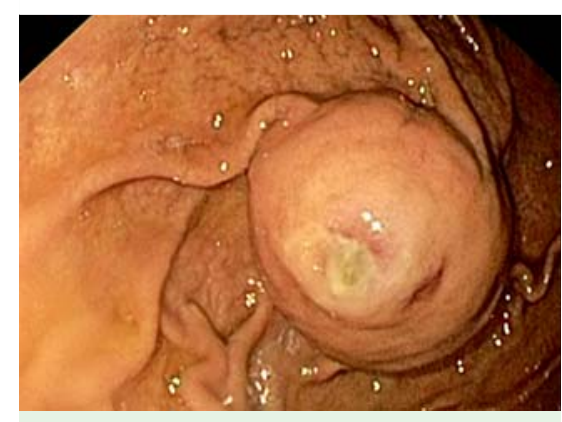

Fig. 1 In a 35-year-old man suffering from Cooley disease, with a history of splenectomy and two recent episodes of hematemesis with severe anemia, emergency esophagogastroduodenoscopy showed a polypoid, centrally ulcerated mass in the gastric fundus. The overlying, nonulcerated mucosa appears normal.

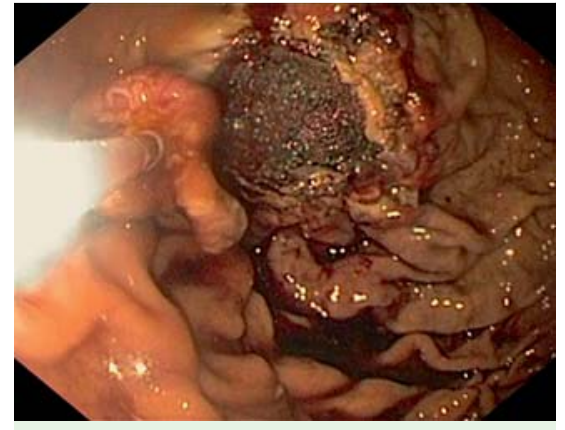

Fig.2 A point during endoscopic resection of the lesion showing the base of the lesion with minimal bleeding.
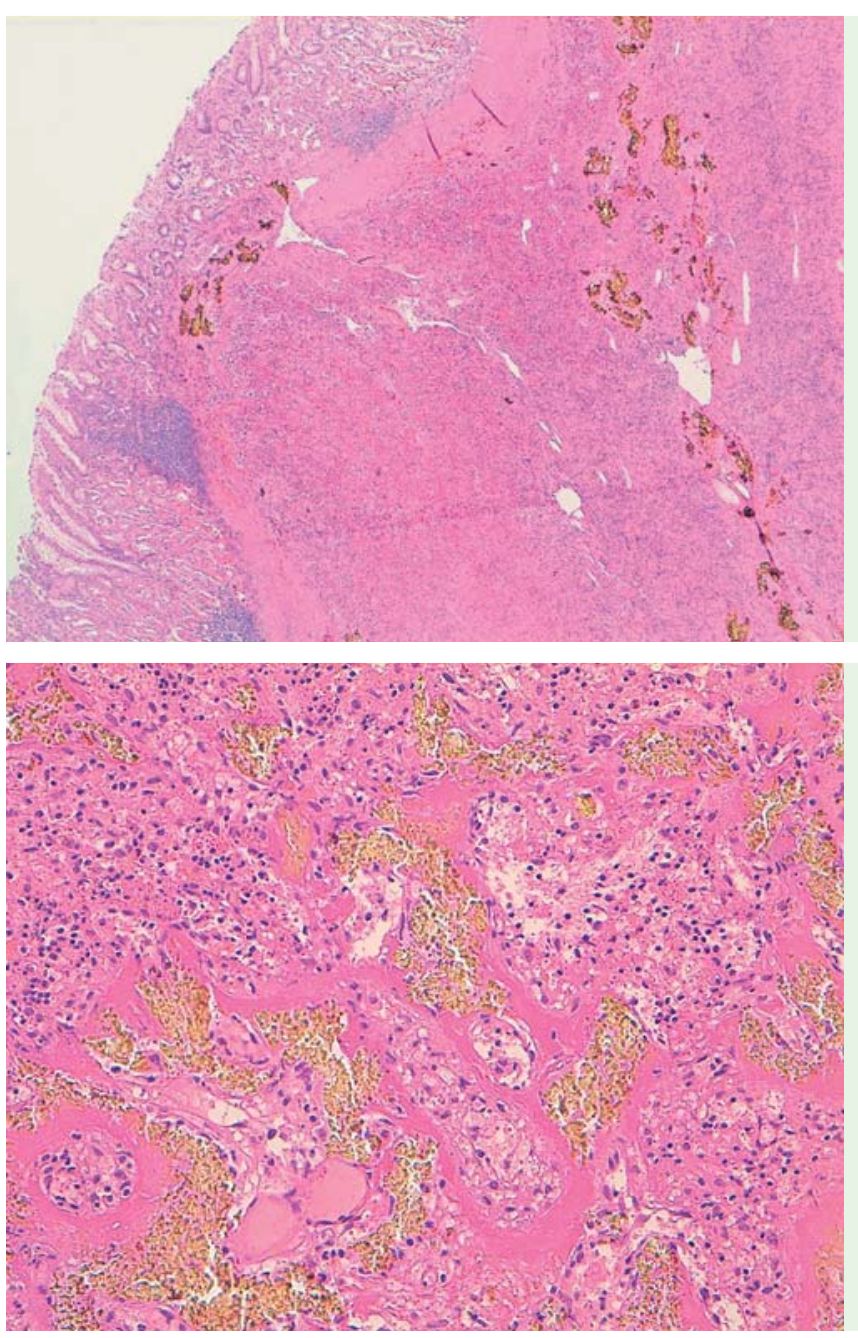

Acute upper gastrointestinal hemorrhage remains a common emergency with annual incidence between 50 and 150 hospital accesses/100000 population/year and a mortality rate of 7-10\% [1]. Endoscopy is the first option both in diagnosis and treatment.

A 35-year-old man, suffering from Cooley disease and with a history of splenectomy, was hospitalized for two recent episodes of hematemesis with severe anemia. Emergency esophagogastroduodenoscopy revealed a protruding, ulcerated $4-\mathrm{cm}$ mass in the gastric fundus; the overlying, nonulcerated, mucosa appeared normal (๑ Fig. 1). Biopsies were not performed because of the risk of bleeding. A computed tomography (CT) scan confirmed the presence of a solid, partially calcified, gastric mass, and endoscopic ultrasound (EUS) showed a rounded, well defined, submucosal hypoechoic lesion. After multidisciplinary discussion, an initial endoscopic approach was decided.

A pre-cut needle was used to create a perilesional perimeter which facilitated the insertion of a diathermic loop. The combined and alternate use of these two instruments enabled precise and complete excision of the entire mass ( Fig.2), despite difficulties as a result of the lesion's intense vascularization and solid consistency. Endoscopic clips were positioned to control two hematic leaks. Histology showed a fibrous and partly calcified mass with pools of erythrocytes and interspersed red and white line cell precursors (๑ Fig.3, Fig.4) corresponding to gastric polypoid extramedullary hematopoiesis.

Extramedullary hematopoiesis is a well described compensatory response to hemoglobinopathies, insufficient medullary hematopoiesis, myelofibrosis and neoplastic replacement, or destruction of the bone marrow. Gastrointestinal localizations are extremely rare and only four cases have been reported either as a single mass [2,3] or multiple localizations $[4,5]$. At 1-month and 6-month follow-up in our patient, the treated region appeared as a retracted scar-like area ( $\bullet$ Fig. 5), and after 3 years, there was complete healing. In conclusion, our study describes the first case of gastric polypoid extramedullary hematopoiesis complicating Cooley disease to be successfully treated with an endoscopic approach.

Endoscopy_UCTN_Code_CCL_1AB_2AD_3AB 


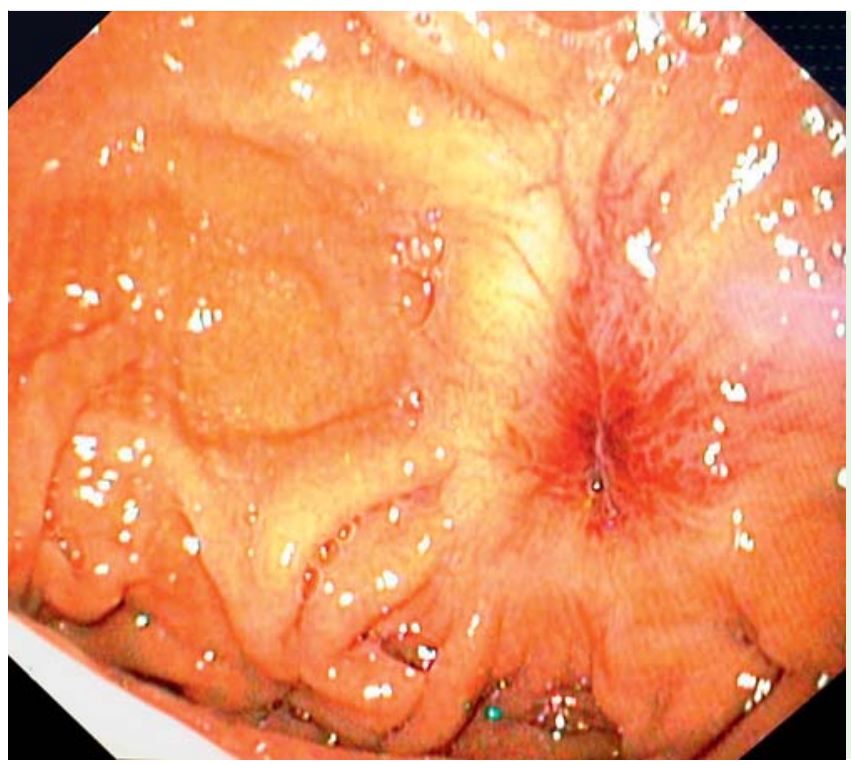

Fig. 5 Follow-up esophagogastroduodenoscopy at 1 month showing a retracted, scar-like area at the site of the earlier endoscopic resection. No lesion or bleeding is seen.

\section{Paola Cognein ${ }^{1, *}$, Valerio Belgrano ${ }^{2, *}$, Luca Mastracci $^{3,4}$, Francesca Pitto ${ }^{3}$, Alberto Fasoli ${ }^{1}$, Franco De Cian ${ }^{2,4}$, Federica Grillo ${ }^{3,4}$}

${ }^{1}$ Gastroenterology and Digestive Endoscopy Unit, IRCCS AOU San Martino IST Genoa, Genoa, Italy

${ }^{2}$ Oncologic Surgery Unit, Department of Surgical Sciences and Integrated Diagnostics (DISC), University of Genoa, Genoa, Italy

${ }^{3}$ Pathology Unit, Department of Surgical Sciences and Integrated Diagnostics (DISC), University of Genoa, Genoa, Italy ${ }^{4}$ IRCCS AOU San Martino IST Genoa, Genoa, Italy

${ }^{*}$ These authors contributed equally to this work.

\section{References}

1 Lau JY, Barkun A, Fan DM et al. Challenges in the management of acute peptic ulcer bleeding. Lancet 2013; 381: 2033 - 2043

2 Gomes AS, Harell GS. Tumefactive extra medullary hematopoiesis of the stomach. Gastrointest Radiol 1976; 1: $163-165$

3 Glew RH, Haese WH, McIntyre PA. Myeloid metaplasia with myelofibrosis: the clinical spectrum of extra medullary hematopoiesis and tumor formation. Johns Hopkins Med J 1973; 132: 253-270

4 Palmer GM, Shortsleeve MJ. Gastric polyps due to extramedullary hematopoiesis. Am J Roentgenol 1998; 171: 531

5 Tiong C, Tai CJ, Chen WY et al. Multiple sessile polypoid lesions in the stomach. Gut 2007; 56: $1754-1769$

\section{Bibliography}

Dol http://dx.doi.org/ 10.1055/s-0034-1390919

Endoscopy 2014; 46: E674-E675

(c) Georg Thieme Verlag KG

Stuttgart · New York

ISSN 0013-726X

Corresponding author Federica Grillo, MD

Pathology Unit

University of Genoa and IRCCS AOU San Martino IST

Department of Surgical Sciences and Integrated Diagnostics (DISC) Largo Rosanna Benzi, 10

Genoa

16132 Italy

Fax: +39-010-5556605

federica.grillo@unige.it 